\title{
Fatores ambientais associados ao tempo de decisão para procura de atendimento no infarto do miocárdio
}

\author{
Environmental factors associated with the decision time for seeking care in myocardial infarction
}

Factores ambientales asociados con el tiempo de decisión para la búsqueda de atención en el infarto de miocardio

\author{
Fernanda Carneiro Mussi', Andreia Santos Mendes', Carla Almeida Damasceno', \\ Mariana de Almeida Moraes Gibaut', Armênio Costa Guimarães", Carlos Antônio de Souza Teles"' \\ ' Universidade Federal da Bahia, Escola de Enfermagem. Salvador-BA, Brasil. \\ "Escola Baiana de Medicina e Saúde Pública. Salvador-BA, Brasil. \\ I"' Universidade Estadual de Feira de Santana, Departamento de Ciências Exatas. Feira de Santana-BA, Brasil.
}

Submissão: 05-10-2012 Aprovação: 24-08-2014

RESUMO

Objetivou-se estimar o tempo de decisão para procura de atendimento (TD) para homens e mulheres com infarto agudo do miocárdio (IAM); analisar a influência de variáveis ambientais no TD e a interação entre gênero e variáveis ambientais para o desfecho TD. Estudo transversal, envolvendo cem pacientes, entrevistados em hospitais de Salvador. Na análise dos dados empregou-se o Qui-quadrado ou Exato de Fisher e o Modelo de Regressão Linear Robusto. Predominou o IAM ocorrido no domicílio, familiares no entorno, e os pacientes sendo alvo de ações equivocadas. Observou-se TD elevado para mulheres $(0,9 \mathrm{~h})$ e homens $(1,4 \mathrm{~h})$. Aqueles em casa no início dos sintomas tiveram maior TD, comparados aos no trabalho e menor em relação aos em via pública $(p=0,047)$. Houve interação estatisticamente significante entre gênero e viver acompanhado; e entre gênero e ter companheiro e filhos no entorno, para o desfecho TD. O cuidar em enfermagem focalizado nas especificidades de fatores ambientais e de gênero pode otimizar o atendimento precoce.

Descritores: Infarto do Miocárdio; Identidade de Gênero; Cuidados de Enfermagem.

\section{ABSTRACT}

The purpose was to estimate the decision time (DT) for searching for attendance for men and women suffering from acute myocardial infarction (AMI); and to analyze the influence of surrounding variables in the DT. Transversal study, involving one hundred patients interviewed in hospitals of Salvador-BA, Brazil. For data analysis, it was used the chi-squared or Fisher's exact test, and the Robust Linear Regression Model. AMI at the home predominated, with family members and patients receiving mistaken actions. A high DT was observed both, for women $(0.9 \mathrm{~h})$ and men (1.4h). Those at home during the initial symptoms had higher DT, compared to those at work; and lower in relation to those in public spaces $(p=0.047)$. Statistically significant interaction occurred among gender and the fact of living with company; and among gender and having a companion and children, for the outcome of the DT. Nursing care focused on the specificity of surrounding factors and gender can optimize early attendance.

Key words: Myocardial Infarction; Gender Identity; Nursing Care.

\section{RESUMEN}

Se objetivó estimar el tiempo de decisión para buscar atendimiento (TD) para hombres y mujeres con infarto agudo de miocardio (IAM); analizar la influencia de variables ambientales en TD y la interacción entre genero y variables ambientales para el desfecho del TD. Estudio transversal, envolviendo cien pacientes entrevistados en hospitales de Salvador-BA, Brasil. En el análisis se utilizó el chi-cuadrado o el Teste Exacto de Fisher y el Modelo de Regresión Linear Robusto. Predominó el IAM en el domicilio, familiares en el entorno y con pacientes siendo objeto de acciones equivocadas. Se observó TD elevados para mujeres $(0,9 \mathrm{~h})$ y hombres $(1,4 \mathrm{~h})$. Aquellos en sus casas en el inicio de los síntomas tuvieron mayor TD, comparados a los en el trabajo, y menor en relación aquellos en vía pública $(p=0,047)$. Hubo interacción estadísticamente significante entre genero y vivir acompañado y entre genero y tener compañero e hijos en el entorno, para el desfecho del TD. El cuidar en enfermería focalizado en especificidades de factores ambientales y de géneros puede optimizar el atendimiento precoce.

Palabras clave: Infarto del Miocardio; Identidad de Género; Atención de Enfermería. 


\section{INTRODUÇÃO}

No Brasil, as doenças cardiovasculares, que incluem o infarto agudo do miocárdio (IAM), permanecem como a primeira causa de mortalidade proporcional, responsável por aproximadamente $32 \%$ dos óbitos ${ }^{(1)}$. Segundo dados do DATASUS ${ }^{(2)}$, no ano de 2009, as doenças cardiovasculares corresponderam a $29 \%$ do total de óbitos no país e, o IAM a 7\% do total de óbitos no referido ano, o que demonstra a relevância desta afecção para a saúde pública. A alta taxa de mortalidade por IAM no Brasil representa um impacto socioeconômico para o país, pois tem retirado indivíduos do mercado de trabalho em plena idade produtiva.

O tratamento de indivíduos com IAM já instalado baseia-se na recuperação do fluxo sanguíneo coronariano, sendo o benefício considerado tempo-dependente ${ }^{(3)}$. Pessoas tratadas na primeira hora de evolução dos sintomas experimentam uma redução significativa da mortalidade hospitalar ${ }^{(4)}$. Assim, o prognóstico depende fundamentalmente da agilidade desses indivíduos para alcançar um serviço de saúde e recuperar a perfusão do miocárdio(3).

No Brasil, há ainda poucos dados disponíveis referentes ao atendimento pré-hospitalar no IAM. E ainda, muitos estudos são inconclusos a respeito dos motivos pelos quais os indivíduos retardam em buscar atendimento diante dos sintomas da doença, especialmente entre os gêneros ${ }^{(5)}$. Estudos nacional ${ }^{(6)} \mathrm{e}$ internacional $^{(7)}$ mostraram que as mulheres com IAM retardaram mais que os homens para decidir procurar um serviço de saúde após o início dos sintomas. Logo, o gênero pode constituir em fator que diferencia o comportamento dos indivíduos face ao IAM, e por isso, merece importância nas investigações.

Estudos têm apontado a influência de variáveis no tempo de decisão para a procura de atendimento face ao IAM, incluindo, as variáveis ambientais ${ }^{(8-9)}$. Todavia, no Brasil é escassa a literatura referente à sua influência no tempo de decisão. Conhecê-la pode orientar práticas em saúde e de enfermagem para a otimização da busca de atendimento precoce.

Diante do exposto, este estudo objetivou estimar o tempo de decisão para procura de um serviço de saúde (TD) para homens e mulheres que sofreram IAM; analisar a influência de variáveis ambientais no TD; e verificar a interação entre gênero e as variáveis para o desfecho TD.

\section{METODOLOGIA}

Trata-se de um estudo exploratório, de corte transversal, realizado em duas instituições hospitalares, referência para o tratamento do IAM, localizadas no município de Salvador-BA.

Para o cálculo do tamanho da amostra (n), tomou-se como parâmetro a prevalência estimada para o infarto agudo do miocárdio (IAM) de 99/100.000 adultos em Salvador/BA ${ }^{(10)}$. Foram também considerados no cálculo da amostra os seguintes parâmetros:

$n=\frac{N P(1-P)}{(N-1) D+P(1-P)}$. onde, $D=\frac{B^{2}}{Z_{\alpha / 2}}$ e $P(|\hat{P}-P| \leq B)=1-\alpha$

$N$ - número de total da população assumida durante o período de coleta de dados $=1.000 ; P$ - proporção dentro da população estudada $=0,099 ; n$ - tamanho da amostra; $\alpha$ - nível de significância; $(1-\alpha) 100 \%$ - grau de confiança; $B$ - erro máximo estimado desejado; $Z_{\alpha / 2}=1,96 ; 1-\alpha=0,95$; $B=0,04$ ou $4 \%{ }^{(11)}$.

De acordo com o cálculo, o tamanho da amostra seria de 99, mas esta foi composta por 100 indivíduos, cujos critérios de inclusão foram: diagnóstico médico de IAM, com ou sem supradesnivelamento do segmento $\mathrm{ST}$, registrado em prontuário; internação há pelo menos $24 \mathrm{~h}$ ou com tempo máximo de pós IAM de 30 dias, para evitar o viés recordatório; orientação no tempo e espaço; ausência de restrições médicas para a entrevista; e aquiescência ao estudo.

O projeto foi aprovado pelo Comitê de Ética das instituições hospitalares, protocolo 11/09, e respeitou os princípios éticos da Resolução 196/96, do Conselho Nacional de Saúde ${ }^{(12)}$.

$\mathrm{O}$ instrumento para a coleta de dados foi constituído por duas partes, com questões estruturadas. A parte I, denominada Dados de caracterização sociodemográfica, constou de: local de internamento, idade, sexo, raça/cor autodeclarada, escolaridade, estado civil, condição laboral, renda familiar, moradores do domicílio, número de dependentes, local de residência e posse de convênio de saúde. A parte II, denominada Componente ambiental do IAM, compreendeu questões referentes ao local de início dos sintomas; dia e hora da decisão pela procura do serviço de saúde; pessoas no entorno ao início dos sintomas; pessoas a quem pediram ajuda, suas ações e o acompanhamento até o serviço de saúde.

A técnica de coleta de dados foi a entrevista, realizada no período de abril a novembro de 2009. Apenas o diagnóstico médico foi confirmado em prontuário. Após a identificação dos indivíduos, no livro de registro e no prontuário, foram selecionados, nas unidades de internação, aqueles que atendiam aos critérios de inclusão, e que estavam livres de exames e/ou possíveis procedimentos por uma hora. Cada participante foi entrevistado uma única vez.

Os dados compuseram uma base de dados do programa SPSS, versão 17.0 for Windows, e foram analisados em percentuais e médias. Para analisar a associação entre as variáveis sociodemográficas e clínicas segundo o gênero empregou-se o teste Qui-quadrado ou o Teste Exato de Fisher na ocorrência de valores esperados abaixo de 5 , em tabelas $2 \times 2$. Nas análises bivariadas e multivariadas, mediante o modelo de Regressão Linear Robusto, verificou-se a associação entre TD e as variáveis ambientais, bem como se testou o termo de interação entre o gênero e as variáveis de interesses. Com a finalidade de corrigir a distribuição da assimetria dos tempos (TD), em decorrência de valores extremos, relatados pelos participantes do estudo, foi aplicada a média geométrica para interpretação do TD ao invés da média aritmética, que tem a propriedade de ser influenciada por valores extremos.

Para a identificação das potenciais variáveis independentes associadas com o desfecho em estudo, considerou-se aquelas que diferiram de zero, sendo estatisticamente significativa ( $p \leq 0,05$, teste bilateral) e no limiar da significância $0,05<p \leq 0,10$, teste bilateral. Esta adoção visou valorizar aquelas associações que seriam descartadas apesar de sua plausibilidade ou importância epidemiológica. 


\section{RESULTADOS}

\section{Caracterização sociodemográfica}

A amostra foi constituída por 100 indivíduos, predominando homens $(71,0 \%)$. A média de idade para os homens $(\mathrm{H})$ foi de $58,70(\mathrm{~S}=11,08)$ anos e para as mulheres $(\mathrm{M})$ de 58,97 $(\mathrm{S}=12,10)$.
Na Tabela 1, verifica-se que a raça/cor autodeclarada mais frequente foi a negra $(71 \%)$, também para $\mathrm{H}$ e M. A maioria da amostra era casada ou tinha união estável $(75,0 \%)$ e essa condição prevaleceu para $\mathrm{H}=58(81,7 \%)$ e $\mathrm{M}=17(58,6 \%)$. A baixa escolaridade foi característica, pois $69,0 \%$ dos indivíduos eram analfabetos, sabiam apenas assinar o nome ou cursaram até o $1^{\circ}$ grau, sendo também prevalente para o grupo de $\mathrm{H}=50(70,3 \%)$ e de $\mathrm{M}=19(65,5 \%)$.

Tabela1 - Caracterização sociodemográfica segundo amostra e gênero, Salvador-BA, 2010

\begin{tabular}{|c|c|c|c|c|c|c|}
\hline \multirow{2}{*}{ FATORES SOCIODEMOGRÁFICOS } & \multicolumn{2}{|c|}{ AMOSTRA } & \multicolumn{2}{|c|}{ HOMENS } & \multicolumn{2}{|c|}{ MULHERES } \\
\hline & $N=100$ & $\%$ & $n=71$ & $\%$ & $\mathbf{n}=\mathbf{2 9}$ & $\%$ \\
\hline \multicolumn{7}{|l|}{ Idade } \\
\hline Menor que 60 anos & 56 & 56,0 & 37 & 52,1 & 19 & 65,5 \\
\hline Maior ou igual a 60 anos & 44 & 44,0 & 34 & 47,9 & 10 & 34,5 \\
\hline \multicolumn{7}{|l|}{ Raça/cor autodeclarada } \\
\hline Branca & 29 & 29,0 & 19 & 26,8 & 10 & 34,5 \\
\hline Negros & 71 & 71,0 & 52 & 73,2 & 19 & 65,5 \\
\hline \multicolumn{7}{|l|}{ Estado civil } \\
\hline Casado(a) & 58 & 58,0 & 46 & 64,8 & 12 & 41,4 \\
\hline Com companheiro(a) & 17 & 17,0 & 12 & 16,9 & 5 & 17,2 \\
\hline Separado(a)/divorciado(a) & 9 & 9,0 & 4 & 5,6 & 5 & 17,2 \\
\hline Solteiro(a) & 9 & 9,0 & 6 & 8,4 & 3 & 10,3 \\
\hline Viúvo(a) & 7 & 7,0 & 3 & 4,2 & 4 & 13,8 \\
\hline \multicolumn{7}{|l|}{ Escolaridade } \\
\hline Analfabeto(a) & 6 & 6,0 & 5 & 7,0 & 1 & 3,4 \\
\hline Assina o nome & 7 & 7,0 & 5 & 7,0 & 2 & 6,9 \\
\hline Até o $1^{\circ}$ grau & 56 & 56,0 & 40 & 56,3 & 16 & 55,2 \\
\hline Até o $2^{\circ}$ grau & 18 & 18,0 & 14 & 19,7 & 4 & 13,8 \\
\hline Até o $3^{\circ}$ grau & 13 & 13,0 & 7 & 9,9 & 6 & 20,7 \\
\hline \multicolumn{7}{|l|}{ Condição laboral } \\
\hline Desempregado(a) & 7 & 7,0 & 4 & 5,6 & 3 & 10,3 \\
\hline Empregado(a) & 14 & 14,0 & 10 & 14,1 & 4 & 13,8 \\
\hline Autônomo(a) & 34 & 34,0 & 22 & 31,0 & 12 & 41,4 \\
\hline Aposentado(a) com atividade & 19 & 19,0 & 17 & 23,9 & 2 & 6,9 \\
\hline Aposentado(a) sem atividade & 26 & 26,0 & 18 & 23,3 & 8 & 27,6 \\
\hline \multicolumn{7}{|l|}{ Renda familiar } \\
\hline Até 3 salários* & 63 & 63,0 & 44 & 62,0 & 19 & 65,5 \\
\hline Acima de 3 salários* & 37 & 37,0 & 27 & 38,0 & 10 & 34,5 \\
\hline \multicolumn{7}{|l|}{$\mathrm{N}^{\circ}$ de dependentes } \\
\hline $0-3$ & 58 & 58,0 & 37 & 52,1 & 21 & 72,4 \\
\hline 3 a 6 & 37 & 37,0 & 31 & 43,7 & 6 & 20,7 \\
\hline$>6$ & 5 & 5,0 & 3 & 4,2 & 2 & 6,9 \\
\hline \multicolumn{7}{|l|}{ Com quem mora } \\
\hline Sozinho(a) & 11 & 11,0 & 8 & 11,3 & 3 & 10,3 \\
\hline Com companheiro(a) & 69 & 69,0 & 54 & 76,1 & 15 & 51,7 \\
\hline Filhos(as) & 63 & 63,0 & 46 & 64,8 & 17 & 58,6 \\
\hline Neto(a) & 23 & 23,0 & 13 & 18,3 & 10 & 34,5 \\
\hline Genro/nora & 15 & 15,0 & 10 & 14,1 & 5 & 17,2 \\
\hline Irmão(a) & 4 & 4,0 & 4 & 5,6 & - & - \\
\hline Outros familiares & 3 & 3,0 & 2 & 2,8 & 1 & 3,4 \\
\hline Amigos(as) & 2 & 2,0 & 2 & 2,8 & - & - \\
\hline Enteado(a) & 2 & 2,0 & 2 & 2,8 & - & - \\
\hline \multicolumn{7}{|l|}{ Convênio } \\
\hline Sim & 1 & 1,0 & - & - & 1 & 3,4 \\
\hline Não & 99 & 99,0 & 71 & 100 & 28 & 96,6 \\
\hline \multicolumn{7}{|l|}{ Local de residência } \\
\hline Salvador & 70 & 70,0 & 51 & 72,2 & 19 & 65,5 \\
\hline
\end{tabular}




\begin{tabular}{lccrrrr}
\hline \multirow{2}{*}{ FATORES SOCIODEMOGRÁFICOS } & \multicolumn{2}{c}{ AMOSTRA } & \multicolumn{2}{c}{ HOMENS } & \multicolumn{2}{c}{ MULHERES } \\
\cline { 2 - 7 } & $\mathbf{N}=\mathbf{1 0 0}$ & $\mathbf{\%}$ & $\mathbf{n = 7 1}$ & $\mathbf{\%}$ & $\mathbf{n = 2 9}$ & $\mathbf{\%}$ \\
\hline Região Metropolitana & 2 & 2,0 & 1 & 1,4 & 1 & 3,4 \\
$\quad$ Outras cidades da Bahia & 28 & 28,0 & 18 & 25,4 & 10 & 31,1 \\
Local de internamento & & & & & \\
$\quad$ Hospital I - HAN & 80 & 80,0 & 57 & 80,2 & 23 \\
Hospital II - HSI & 20 & 20,0 & 14 & 19,8 & 6 & 67,6 \\
\end{tabular}

*Salário mínimo no período de coleta de dados: $R \$ 465,00$.

No que tange à atividade laboral, $67,0 \%, \mathrm{H}=49(69,0 \%)$ e $M=18(62,1 \%)$, eram profissionalmente ativos, ou seja, empregados(as), autônomos(as) ou aposentados(as) com atividade; $33,0 \%, H=22(28,9 \%)$ e $M=11(37,9 \%)$, não tinham atividade profissional por estarem desempregados ou aposentados sem atividade. A renda familiar predominante foi de até três salários mínimos tanto para os $\mathrm{H}=44(62,0 \%)$ quanto para as $\mathrm{M}=19(65,5 \%)$.

$\mathrm{O}$ número de dependentes prevalente foi de até três (58\%), o mesmo sendo observado para os $\mathrm{H}=37(52,1 \%)$ e as $M=21(72,4 \%)$. Com relação ao número de pessoas com quem dividiam a residência, $11 \%$ moravam sozinhos e, os demais $89 \%$, com outros familiares ou amigos, sobressaindo-se entre eles o(a) companheiro(a) (69\%) e filhos(as) (63\%), perfazendo uma média de duas pessoas por moradia. Os $\mathrm{H}$ em relação às $M$ viviam mais com a companheira $(p=0,017)$ e as $M$ com suas netas $(p=0,081)$.

Os participantes foram predominantemente procedentes de Salvador e Região Metropolitana (72,0\%). Com relação ao hospital de internamento, $80,0 \%$ foram internados no Hospital I e $20 \%$ no Hospital II. A amostra foi atendida pelo Sistema Único de Saúde (SUS), apesar de uma participante $(1,0 \%)$ ter referido posse de convênio de saúde.

\section{Caracterização do contexto ambiental no início do infarto agudo do miocárdio}

$\mathrm{Na}$ Tabela 2, verifica-se que mais da metade da amostra $(76,0 \%)$ estava em casa quando os sintomas do IAM começaram, o mesmo sendo observado para os gêneros $(p=0,580)$.

Tabela 2 - Caracterização do contexto ambiental segundo amostra e gênero, Salvador-BA, 2010

\begin{tabular}{|c|c|c|c|c|c|c|c|}
\hline \multirow{2}{*}{ CARACTERÍSTICAS AMBIENTAIS } & \multicolumn{2}{|c|}{ AMOSTRA } & \multicolumn{2}{|c|}{ HOMENS } & \multicolumn{2}{|c|}{ MULHERES } & \multirow{2}{*}{$\begin{array}{l}\text { Valo } \\
\text { de } p\end{array}$} \\
\hline & $\mathbf{n}$ & $\%$ & $\mathbf{n}$ & $\%$ & $\mathbf{n}$ & $\%$ & \\
\hline Local do início dos sintomas $(n=100)$ & 100 & & 71 & & 29 & & \\
\hline Casa & 76 & 76,0 & 52 & 73,2 & 24 & 86,2 & 0,580 \\
\hline Via pública & 18 & 18,0 & 14 & 18,3 & 4 & 10,3 & \\
\hline Trabalho & 6 & 6,0 & 5 & 7,0 & 1 & 3,4 & \\
\hline Pessoas no entorno no início dos sintomas & 68 & & 45 & & 23 & & \\
\hline Companheiro(a) & 38 & 55,9 & 30 & 66,7 & 8 & 34,8 & 0,170 \\
\hline Filhos(as) & 23 & 33,8 & 12 & 26,7 & 11 & 47,8 & 0,024 \\
\hline Outros familiares & 17 & 25,0 & 12 & 26,7 & 5 & 21,7 & 0,971 \\
\hline Colegas de trabalho/vizinhos & 8 & 11,8 & 5 & 11,1 & 3 & 13,0 & 0,573 \\
\hline Pessoas solicitadas para ajuda & 90 & & 63 & & 27 & & \\
\hline Companheiro(a) & 39 & 43,3 & 31 & 49,2 & 8 & 29,6 & 0,134 \\
\hline Filhos(as) & 31 & 34,4 & 20 & 31,7 & 11 & 40,7 & 0,341 \\
\hline Outros familiares & 18 & 20,0 & 14 & 22,2 & 4 & 14,8 & 0,485 \\
\hline Vizinhos & 16 & 17,8 & 12 & 19,0 & 4 & 14,8 & 0,701 \\
\hline Colegas de trabalho & 4 & 4,4 & 2 & 3,1 & 2 & 7,4 & 0,341 \\
\hline Ações das pessoas do entorno & 41 & & 25 & & 16 & & \\
\hline Disseram para buscar um serviço de saúde/ajuda/realizar exames & 25 & 60,1 & 15 & 60,0 & 10 & 62,5 & 0,956 \\
\hline Ofereceram alguma coisa para tomar & 11 & 26,8 & 7 & 28,0 & 4 & 25,0 & 0,571 \\
\hline $\begin{array}{l}\text { Não deram importância/Disseram para não de preocupar / } \\
\text { Orientaram a descansar }\end{array}$ & 5 & 12,2 & 3 & 12,0 & 2 & 12,5 & 0,342 \\
\hline Acompanhante até o $1^{\circ}$ local de atendimento & 86 & & 60 & & 26 & & \\
\hline Vizinhos(as) & 40 & 46,5 & 25 & 41,7 & 15 & 57,7 & 0,126 \\
\hline Filhos(as) & 33 & 38,3 & 26 & 43,3 & 7 & 26,9 & 0,228 \\
\hline Outros familiares & 19 & 22,1 & 13 & 21,7 & 6 & 23,0 & 0,781 \\
\hline Companheiro(a) & 10 & 11,6 & 8 & 13,3 & 2 & 7,7 & 0,506 \\
\hline Colegas de trabalho/amigos & 5 & 5,8 & 4 & 6,7 & 1 & 3,8 & 0,873 \\
\hline
\end{tabular}


A maioria da amostra $(68,0 \%)$ estava em companhia de alguém no início do evento cardiovascular. A média de acompanhantes por participantes foi de 2,2. Dentre as pessoas no entorno predominaram o(a) companheiro(a) (55,9\%) e os(as) filhos(as) (33,8\%). Na análise por gênero, verificou-se que os H estavam em maior proporção sozinhos (36,6\%), em relação às mulheres $(21,7 \%),(p=0,486)$. Quando acompanhados, os $\mathrm{H}$ estavam em maior proporção com a companheira, do que as mulheres $(p=0,170)$. As $M$ estavam em maior proporção acompanhadas pelos filhos do que os homens e essa associação foi estatisticamente significante $(p=0,024)$.

Dos 100 participantes, 90 pediram ajuda a alguém face aos sintomas do IAM predominando a figura do(a) companheiro(a) $(44,3 \%)$ seguida dos filhos(as) (34,3\%). As M clamaram com maior frequência por seus filhos(as), em seguida por seus companheiros, e os $\mathrm{H}$ por suas companheiras, seguido dos(as) filhos(as). No entanto, não houve associação entre pessoas evocadas para ajuda e o gênero.

Dos 90 participantes que estavam acompanhados, 41 foram alvo de suas ações. Predominaram para a amostra, e para $\mathrm{H}$ e $\mathrm{M}$, as ações de "dizer para procurar atendimento médico/pedir ajuda/realizar exames" (60,1\%; $\mathrm{H}=60,0 \%$ e $M=62,5 \%)$, seguidas de "oferecer algo para tomar" $(26,8 \%$; $H=28,0 \%$ e $M=25,0 \%$ ). Menor proporção de participantes não tiveram suas queixas valorizadas pelas pessoas no entorno em razão de ações como "não dar importância", "dizer para não se preocupar e orientar a descansar" $(12,2 \%$; $H=12,0 \%$ e $M=12,5 \%$ ).

Predominaram como acompanhantes dos participantes até o serviço de saúde, tanto para a amostra como para os gêneros, os vizinhos $(46,5 \%)$ seguidos dos(as) filhos(as) $(38,3 \%)$, outros familiares $(22,1 \%)$ e companheiro(a) $(11,6 \%)$. Não houve associação entre gênero e tipo de acompanhante ao local de atendimento médico.

\section{Comparação dos tempos de decisão segundo fatores am- bientais e o gênero}

A média geométrica (MG) e a mediana do TD foram, respectivamente, $1,1 \mathrm{~h}$ e $1,0 \mathrm{~h}$. Para as $\mathrm{M}$, a MG para TD foi de $1,4 \mathrm{~h}$ e a mediana de $1,5 \mathrm{~h}$ e, para os $\mathrm{H}$, a MG foi de $0,9 \mathrm{~h}$ e a mediana de 1,0h. As mulheres apresentaram tempos médios de decisão maiores do que os $\mathrm{H}$, no entanto não houve diferença estatisticamente significante.

$\mathrm{Na}$ Tabela 3 observa-se que as pessoas que estavam em casa no início dos sintomas tiveram maior TD em relação às que estavam em via pública e menor TD dos que estavam no trabalho $(p=0,047)$. Não houve interação entre gênero e estar em casa no início dos sintomas para o desfecho TD.

Tabela 3 - Características do contexto ambiental associados ao TD e o gênero, Salvador-BA, 2010

\begin{tabular}{|c|c|c|c|c|c|}
\hline \multirow[b]{2}{*}{ CARACTERÍSTICAS DO CONTEXTO AMBIENTAL } & \multirow{2}{*}{$\begin{array}{c}\text { MG TD* } \\
\text { AMOSTRA } \\
\mathbf{n}=\mathbf{1 0 0}\end{array}$} & \multirow[b]{2}{*}{$\mathrm{p}+$} & \multicolumn{2}{|c|}{ MG TD* } & \multirow[b]{2}{*}{ pł } \\
\hline & & & $\mathrm{H} n=71$ & $M n=29$ & \\
\hline $\begin{array}{l}\text { Local do início dos sintomas } \\
\text { Casa (76) } \\
\text { Trabalho (6) } \\
\text { Via pública (18) }\end{array}$ & $\begin{array}{l}1,3 \\
1,4 \\
0,4\end{array}$ & 0,047 & $\begin{array}{l}1,2 \\
1,1 \\
0,4\end{array}$ & $\begin{array}{l}1,6 \\
4,0 \\
0,5\end{array}$ & 0,977 \\
\hline $\begin{array}{l}\text { Pessoas do entorno no início dos sintomas } \\
\text { Sim (68) } \\
\text { Não (32) }\end{array}$ & $\begin{array}{l}0,7 \\
1,3\end{array}$ & 0,119 & $\begin{array}{l}0,7 \\
1,1\end{array}$ & $\begin{array}{l}0,6 \\
1,8\end{array}$ & 0,486 \\
\hline $\begin{array}{l}\text { Companheiro(a) } \\
\text { Sim (38) } \\
\text { Não }(62)\end{array}$ & $\begin{array}{l}1,5 \\
0,9\end{array}$ & 0,165 & $\begin{array}{l}1,1 \\
0,9\end{array}$ & $\begin{array}{l}6,1 \\
0,8\end{array}$ & 0,012 \\
\hline $\begin{array}{l}\text { Filhos (as) } \\
\text { Sim (23) } \\
\text { Não (77) }\end{array}$ & $\begin{array}{l}0,8 \\
1,2\end{array}$ & 0,373 & $\begin{array}{l}0,4 \\
1,2\end{array}$ & $\begin{array}{l}1,8 \\
1,2\end{array}$ & 0,106 \\
\hline $\begin{array}{l}\text { Vizinhos (as) } \\
\text { Sim (3) } \\
\text { Não (97) }\end{array}$ & $\begin{array}{l}1,3 \\
1,1\end{array}$ & 0,791 & $\begin{array}{l}2,6 \\
0,9\end{array}$ & $\begin{array}{l}0,3 \\
2,5\end{array}$ & 0,985 \\
\hline $\begin{array}{l}\text { Colegas de trabalho } \\
\text { Sim (5) } \\
\text { Não (95) }\end{array}$ & $\begin{array}{l}1,6 \\
1,0\end{array}$ & 0,474 & $\begin{array}{l}1,6 \\
0,9\end{array}$ & $\begin{array}{l}1,7 \\
1,4\end{array}$ & 0,762 \\
\hline $\begin{array}{l}\text { O que as pessoas no entorno disseram para fazer } \\
\text { Ofereceram algo para tomar } \\
\text { Sim (11) } \\
\text { Não (89) }\end{array}$ & $\begin{array}{l}1,9 \\
0,9\end{array}$ & 0,100 & $\begin{array}{l}2,0 \\
0,8\end{array}$ & $\begin{array}{l}1,9 \\
1,3\end{array}$ & 0,440 \\
\hline $\begin{array}{l}\text { Não deram importância } \\
\text { Sim (5) } \\
\text { Não (95) }\end{array}$ & $\begin{array}{l}4,2 \\
1,0\end{array}$ & 0,117 & $\begin{array}{l}5,6 \\
0,9\end{array}$ & $\begin{array}{l}2,8 \\
1,3\end{array}$ & 0,116 \\
\hline $\begin{array}{l}\text { Disseram para buscar atendimento médico } \\
\text { Sim }(25) \\
\text { Não }(75)\end{array}$ & $\begin{array}{l}1,9 \\
0,9 \\
\end{array}$ & 0,680 & $\begin{array}{l}0,8 \\
0,7\end{array}$ & $\begin{array}{l}1,7 \\
1,3\end{array}$ & 0,571 \\
\hline
\end{tabular}

*MG - Média Geométrica, em horas, do TD;

p†-mostra a diferença entre as categorias das variáveis específicas;

pł- mostra a heterogeneidade entre homens e mulheres (a interação).

Todos os valores de $p$ foram obtidos pelo modelo de regressão linear robusto. 
Não se observou associação significante entre haver ou não pessoas no entorno no início dos sintomas e TD. Verificou-se interação entre gênero e ter o companheiro como pessoa no entorno para o desfecho TD $(p=0,012)$ e ter filhos(as) no entorno para o desfecho TD $(p=0,106)$. As $M$ que estavam com companheiros e filhos(as), em relação aos $\mathrm{H}$ com as companheiras e filhos(as) tiveram maior TD $(6,1 \mathrm{~h}$ e $1,8 \mathrm{~h})$.

Os participantes que sofreram a ação das pessoas no entorno não apresentaram maior TD. Não constatou-se também interação entre gênero e ação das pessoas no entorno para o desfecho TD.

\section{DISCUSSÃO}

Neste estudo predominaram homens com IAM o que corresponde aos achados na literatura internacional ${ }^{(8)} \mathrm{e}$ nacional ${ }^{(6)}$. As mulheres desenvolveram IAM mais precocemente considerando-se as idades limites de 55 anos para os homens e 65 anos para as mulheres, tomadas como ponto de corte para a prematuridade de IAM em relação aos gêneros ${ }^{(13)}$. A amostra apresentou também características sociodemográficas homogêneas, pois era usuária do SUS, oriunda basicamente da cidade de Salvador e Região Metropolitana, declarando-se majoritariamente de raça/cor negra e vivendo em condição de desigualdade social evidenciada pela baixa escolaridade e renda familiar. Estes achados confirmam as descrições na literatura nacional ${ }^{(14)} \mathrm{e}$ internacional ${ }^{(15)}$ dessas características como potencializadoras do risco de doença cardiovascular.

Tanto os homens como as mulheres retardaram para decidir procurar um serviço de saúde, as mulheres apresentando tempo superior $(1,4 \mathrm{~h} v s 0,9 \mathrm{~h})$, o que expôs os gêneros a elevado risco de mortalidade, pois cerca de $50 \%$ das pessoas com IAM morrem na primeira hora de evolução do evento cardiovascular $^{(4)}$. Há que se considerar que após a tomada de decisão os participantes ainda despenderam tempo deslocando-se para um serviço de saúde até obter o diagnóstico e tratamento.

Outras investigações também ${ }^{(6-7)}$ verificaram que as muIheres com IAM, comparadas aos homens, retardaram mais para procurar um serviço de saúde após o início dos sintomas. Desta forma, o gênero pode ser um fator que diferencia o comportamento de indivíduos quanto a doença e o uso dos serviços de saúde. Muitas vezes, as mulheres não querem incomodar com seus problemas de saúde e demoram em procurar atendimento ${ }^{(16)}$. A possibilidade de afastamento pela doença pode provocar o medo da desorganização familiar, do desamparo da família ao deixar seus membros sem o cuidado costumeiramente dispensado e, ainda, o medo da dependência econômica ou física ${ }^{(17)}$. Assim, a resistência em buscar por atendimento configura-se como uma tentativa de preservar a vida cotidiana para desempenhar, principalmente, o papel de organizadora do espaço doméstico ${ }^{(18)}$. Nesse sentido, percebe-se a importância dos trabalhos de educação em saúde envolvendo as questões de gênero, no intuito de minimizar os comportamentos individuais que são prejudiciais a saúde.

Apesar de não ter ocorrido interação entre o local de início dos sintomas do IAM e gênero para o desfecho TD, observou-se que as mulheres estavam majoritariamente em casa. Já os homens estavam em maior proporção em via pública e no trabalho. Tais achados parecem refletir ainda os papéis sociais dos homens como provedores da família e das mulheres como mães e organizadoras do espaço doméstico. Vale destacar que homens e mulheres inserem-se na vida familiar segundo referenciais de gênero, apreendidos ao longo da vida e que determinam funções socialmente legitimadas ${ }^{(19)}$.

Ainda no tocante ao local de início dos sintomas do evento cardiovascular, observou-se que a maioria dos participantes estava em casa. Esses tiveram maior TD em relação aos que estavam em via pública e menor TD em relação aos que estavam no trabalho no início dos sintomas. Parece que o mundo do trabalho ofereceu maior resistência a tomada de decisão e que os locais de trabalho dos participantes do estudo não estavam preparados para ajudá-los a decidir pela procura imediata de atendimento. As pessoas em via pública chegaram mais rápido, e isso talvez se deva a sensibilidade para ajuda daqueles em seu entorno no espaço público. Esses achados contrastam com os resultados de outras pesquisas, que apontaram o estar no domicílio como fator contribuinte para maior TD em relação a outros locais ${ }^{(9)}$. Tal estudo ressaltou que os indivíduos que estavam em casa resistiram mais aos sintomas ou entraram em contato com o próprio médico ao invés de procurar o serviço de emergência e, portanto, foram susceptíveis a maior tempo de decisão para a procura do atendimento médico ${ }^{(9)}$.

A maioria dos participantes estava acompanhada no início dos sintomas, especialmente por familiares, e esses apresentaram menor TD em relação aos que estavam sozinhos, embora sem associação estatisticamente significante. De modo semelhante, em outro estudo ${ }^{(9)}$, os indivíduos cujos sintomas começaram na presença de alguém tiveram menor TD. Já foi constatado $^{(20)}$ que familiares e vizinhos julgaram a gravidade dos sintomas do evento cardiovascular em curso e decidiram pela procura de ajuda. Portanto, aqueles presentes no contexto ambiental do IAM podem influenciar e auxiliar na busca de atendimento, combatendo as ações de resistência por parte das mesmas. É preciso salientar que as vítimas de IAM podem resistir à procura de um serviço de saúde, alimentando a esperança de melhora e de que nada mais grave estaria acontecendo ou, mesmo, podem se sentir dominadas pelos sintomas e, portanto, sem condições para acionar um serviço médico de emergência.

Estes achados revelam a importância de familiares e da rede de vizinhança, de pessoas em risco potencial para IAM, serem alvos de programas educativos nos serviços de saúde que frequentam, visando à redução dos tempos de retardo pré-hospitalar e a implementação de ações de suporte básico de vida face ao IAM. A capacitação desses grupos para o reconhecimento dos sintomas e estar à frente na decisão e no acionamento de um serviço de emergência, pode evitar ações equivocadas como a de relativizar a gravidade da situação e de oferecer líquidos, a exemplo de chá, água entre outros, tal como constatado no presente estudo. As ações equivocadas das pessoas no entorno determinaram maior TD. 
Depreende-se, portanto, que ter uma pessoa no entorno contribuiu para menor TD, todavia algumas ações realizadas por essas pessoas podem contribuir para o aumento ou diminuição do TD, a depender da sua adequação ao IAM.

Embora os participantes acompanhados no início dos sintomas tenham apresentado menor TD em relação aos que estavam sozinhos, verificou-se interação entre gênero e pessoas no entorno para o desfecho TD. As mulheres na presença de companheiros e filhos(as), em relação aos homens com companheiras e filhos(as), apresentaram maior TD. Tais achados sugerem a forte representação materna no grupo estudado. É possível que as queixas das mulheres sejam menos valorizadas no contexto familiar e o fato de naturalmente estarem imbuídas das responsabilidades do lar e da preocupação com cuidados de seus familiares pode fazer com que sejam relativizadas as suas próprias necessidades por atendimento em saúde. Constatou-se também que as pessoas mais frequentemente no entorno de vítimas de IAM foram solicitadas para ajuda, sendo que as mulheres clamaram com maior frequência por seus filhos(as) e os homens por suas companheiras. Corroborando com esses achados outro estudo(21) mostrou que os homens com IAM foram mais encorajados pelas esposas a ir ao hospital ou acionar uma ambulância, já as esposas foram mais encorajadas por seus filhos do que pelos maridos.

Com relação ao acompanhante até o primeiro local procurado para atendimento, houve predominância de vizinhos, seguidos de filhos(as), outros familiares, companheiro(a) e colegas de trabalho. Apesar de não ter ocorrido associação entre o gênero e o tipo de acompanhante, predominaram os vizinhos para as mulheres e os(as) filhos(as) para os homens, provavelmente em virtude de fatores como disponibilidade de tempo, proximidade geográfica, rede de suporte social ou posse de transporte particular para a condução da vítima.

Programas de educação comunitária precisam abranger diferentes grupos, incluindo empresas, escolas, bases militares, complexos habitacionais, prédios públicos, grupos religiosos, centros de saúde e instituições hospitalares, programas de saúde da família, etc. Devem visar aumentar a porcentagem de pessoas que chamam o serviço de emergência e obtêm ajuda rapidamente. Públicos alvo e enfermeira(o)s devem trabalhar juntos para ajudar a reduzir o intervalo de tomada de decisão entre o início dos sintomas e a iniciativa de procurar socorro.

Junto à equipe de saúde $\mathrm{a}(\mathrm{o})$ enfermeira(o) deve enfrentar o desafio de descobrir populações de maior risco e recrutar familiares dos pacientes para se tornarem peritos conscientes, modelos de entusiasmo e de motivação e, também, intervencionistas bem informados e treinados. E, considerando que o infarto está associado a populações de baixa renda e nível de instrução, como constatado nessa investigação, esses grupos devem receber cobertura especial dos programas educativos, bem como de ações pedagógicas compatíveis com suas possibilidades de aprendizagem. Além disso, programas de treinamento continuado devem ser desenvolvidos para prevenir a deterioração das habilidades necessárias. A(o) enfermeira(o) deve ocupar espaço importante na prevenção, reconhecendo-se como sujeito que pode contribuir no processo educativo de indivíduos, visando a conscientização dos sinais de eventos cardiovasculares iminentes, a execução de condutas iniciais frente a esses e a otimização da busca imediata por um serviço de saúde diante dos sinais e sintomas prodrômicos do IAM $^{(18)}$.

Tais programas devem privilegiar uma abordagem diferenciada para homens e mulheres, já que seus comportamentos, face aos sintomas do IAM, podem ser influenciados pela perspectiva social de gênero. $\mathrm{A}(\mathrm{o})$ enfermeira(o) pode atuar no plano simbólico dos indivíduos, com abordagem de gênero, visando desenvolver a percepção de que resistir aos sintomas pode significar o risco de perder a vida ou limitá-la permanentemente ${ }^{(18)}$.

\section{CONSIDERAÇÕES FINAIS}

O estudo trouxe contribuições sobre os fatores ambientais e de gênero que interferem no TD dos indivíduos com IAM para a procura de um serviço de saúde, na amostra estudada em Salvador/BA.

Observou-se que a maioria dos participantes estava no domicílio e com familiares no entorno no início do IAM. A maioria pediu ajuda a companheiros, filhos, outros familiares e vizinhos e foi alvo de várias ações equivocadas.

Observou-se TD elevados para homens e mulheres, essas apresentando tempos superiores, mas sem diferença estatisticamente significante. Encontrou-se associação significante para as pessoas em casa no início dos sintomas (TD =1,3h), em relação as que estavam no trabalho (TD=1,4h) ou via pública (TD $=0,3 \mathrm{~h}$ ). Houve interação entre gênero e viver acompanhado e entre gênero e ter companheiro e filhos como pessoas no entorno, para o desfecho TD. As mulheres na presença de companheiros e filhos(as), em relação aos homens com companheiras e filhos(as), apresentaram maior TD.

O conhecimento obtido oferece subsídios para práticas de cuidar em enfermagem focalizadas nas especificidades desses fatores e dos gêneros, vislumbrando intervenções que promovam a ação correta e a valorização da busca por atendimento em saúde por pessoas que sofrem IAM.

Destaca-se como possível limitação do estudo o tamanho da amostra. É possível aventar a hipótese de que, com amostras maiores, outras associações significantes poderiam ter sido encontradas. Cabe também ser considerado que o presente trabalho deteve-se a verificar a interação entre TD e as variáveis ambientais, segundo o gênero e, outras variáveis, a exemplo das clínicas, cognitivas e emocionais precisam ser avaliadas em outras pesquisas.

O campo de análise com base na categoria gênero é ainda incipiente, necessita de aprofundamento por meio de pesquisas que poderão trazer benefícios para a prevenção da morbidade e mortalidade pela doença. Intervenções educacionais em campanhas públicas ou individualizadas que focalizem as diferenças específicas de gênero, identificadas no presente estudo, precisam ser testadas em comunidades e pessoas com maior risco para IAM. 


\section{REFERÊNCIAS}

1. Escosteguy $\mathrm{CC}$ et al. Implementando diretrizes clínicas na atenção ao infarto agudo do miocárdio em uma emergência pública. Arq Bras Cardiol [Internet]. 2011 [acesso em 05 de outubro de 2012];96(1):18-25. Disponível em: http://www.scielo.br/scielo.php?pid=S0066-782 X2011000100005\&script $=$ sci_arttext

2. Ministério da Saúde. Indicadores de Saúde. Datasus. [Internet]. [S.I]: Ministério de Saúde [acesso em 05 de outubro de 2012]. Disponível em: http://www2.datasus.gov. br/DATASUS/index.php?area $=0201$

3. Pesaro AEP, Serrano CVJ, Nicolau JC. Infarto agudo do miocárdio: síndrome coronariana aguda com supradesnível do segmento ST. Rev Assoc Med Bras [Internet]. 2004 [acesso em 05 de outubro de 2012];50(2):214-20. Disponível em: http://www.scielo.br/pdf/ramb/v50n2/20786.pdf

4. Sociedade Brasileira de Cardiologia. III Diretriz sobre tratamento do infarto agudo do miocárdio. Arq Bras Cardiol [Internet]. 2004 [acesso em 05 de outubro de 2012];83(Suppl. 4):9-86. Disponível em: http://publicacoes.cardiol.br/consenso/2004/Dirlll_TratalAM.pdf

5. Devon HA, Zerwic JJ. Symptoms of acute coronary syndormes: are there gender differences? A review of the literature. Heart Lung [Internet]. 2002 [cited 2012 October 05];31(4):235-45. Available from: http://www.ncbi.nlm. nih.gov/pubmed/12122387

6. Sampaio ES. Fatores relacionados ao retardo pré-hospitalar de pessoas com infarto agudo do miocárdio [dissertação]. Salvador (BA): Universidade Federal da Bahia; 2008.

7. Moser DK, Mckinley S, Dracup K, Chung ML. Gender differences in reasons patients delay in seeking treatment for acute myocardial infarction synptoms. Patient Education Couns [Internet]. 2005 [cited 2012 October 05];(56):45-54. Disponível em: http://www.ncbi.nlm.nih. gov/pubmed/15590222

8. Gärtner C, Walz L, Bauernschmitt E, Ladwig KH. The causes of prehospital delay in myocardial infarction. Dtsch Arztebl Int [Internet]. 2008 [cited 2012 October 05];105(15):286-91. Available from: http://www.ncbi. nlm.nih.gov/pmc/articles/PMC2696860/

9. Perkins-Porras L, Whitehead DL, Strike PC, Steptoe A. Pre-hospital delay in patients with acute coronary syndrome: factors associated with patient decision time and home-to-hospital delay. Eur J Cardiovasc Nurs [Internet]. 2009 [cited 2012 October 05];8(1):26-33. Available from: http://www.ncbi.nlm.nih.gov/pubmed/18635400

10. Lessa I. Epidemiologia das doenças cerebrovasculares no Brasil. Rev ROCESP. 1999;9(4):509-18.

11. Kish L. Survey Sampling. New York (US): Witley; 1965.
12. Ministério da Saúde (BR), Conselho Nacional de Saúde. Resolução $n^{\circ}$. 196, de 10 de outubro de 1996. Sobre a pesquisa envolvendo seres humanos [Internet]. Diário Oficial da União 1996 [acesso em 10 de setembro 2012]. Disponível em: conselho.saude.gov.br/resolucoes/1996/ reso196.doc

13. Conti RAS, Solimene MC, Luz PL, Benjá AM, Lemos Neto PA, Ramires JAF. Comparação entre homens e mulheres jovens com infarto agudo do miocárdio. Arq Bras Cardiol. 2002;79(5):510-7.

14. Ishitani LH, Franco GC, Perpétuo IH, França E. Desigualdade social e mortalidade precoce por doenças cardiovasculares no Brasil. Rev Saúde Pública. 2006;40:684-91.

15. Yusuf S, Reddy S, Ounpuu S, Anand S. Global burden of cardiovascular disease: part II variations in cardiovascular disease by specific ethnic groups and geographic regions and prevention strategies. Circulation [Internet]. 2001 [cited 2012 October 05];104(23):2855-64. Available from: http://www.ncbi.nlm.nih.gov/pubmed/11733407

16. Kristofferzon ML, Löfmark R, Carlsson M. Myocardial infarction: gender differences in coping and social support. J Adv Nurs. 2003;44(4):360-74.

17. Mussi FC, Ferreira SL, Menezes AA. Vivências de mulheres à dor no infarto do miocárdio. Rev Esc Enferm USP [Internet]. 2006 [acesso em 05 de outubro de 2012];40(2):1708. Disponível em: http://www.scielo.br/scielo.php?pid= S0080-62342006000200004\&script =sci_arttext

18. Mussi FC, Pereira A. Tolerância à dor no infarto do miocárdio. Acta Paul Enferm [Internet]. 2010;23(1):80-7. Disponível em: http://www.scielo.br/scielo.php?pid=S0103$-21002010000100013 \&$ script $=$ sci_arttext

19. Freitas WMF, Silva ATMC, Coelho EAC, Guedes RN, Lucena KDT, Costa AT. Paternidade: responsabilidade social do homem no papel de provedor. Rev Saúde Pública [lnternet]. 2009 [acesso em 05 de outubro de 2012];43(1):8590. Disponível em: http://www.scielo.br/scielo.php?pid= S0034-89102009000100011\&script = sci arttext

20. Pattenden J, Watt I, Lewin RJP, Stanford N. Decision making processes in people with symptoms of acute myocardial infarction: qualitative study. BMJ [Internet]. 2002 [cited 2012 October 05];324:1-5. Available from: http:// www.bmj.com/content/324/7344/1006

21. Thuresson $M$, Jarlov MB, Lindhal B, Svensson $L$, Zedigh C, Herlitz J. Thoughts, actions, and factores associated with prehospital delay in patients with acute coronary syndrome. Heart Lung [Internet]. 2007 [cited 2012 October 05];36(6):398-409. Available from: http://www.ncbi. nlm.nih.gov/pubmed/18005801 